\title{
Current and emerging treatment options for pediatric eosinophilic esophagitis
}

This article was published in the following Dove Press journal:

Pediatric Health, Medicine and Therapeutics

30 August 2013

Number of times this article has been viewed

\section{Yoram Elitsur \\ Department of Pediatrics, Gastroenterology Division, Marshall University, John C Edwards School of Medicine, Huntington, WV, USA}

\begin{abstract}
Eosinophilic esophagitis is a new clinicopathologic disorder and has been recognized as a chronic disease in children and adults. The disease is associated with environmental and food allergies, but the exact pathophysiology has not yet been discovered. Epidemiological studies showed a global distribution of the disease and an uneven geographic distribution within the United States where the northeastern states had a higher rate of prevalence compared to the western states. Studies have shown that there are multiple confounding factors that affect the phenotypic characteristics of the disease which may include place of residence (rural versus urban), ethnicity, race, and genetics, among others. Clinical presentation may be different between adult and pediatric patients, but the endoscopic and histological characteristics of the disease are very similar between those groups. The pathophysiology of the disease has not yet been established, but the mucosal immune system, especially the pathway involving the eosinophil lineage, has been suggested as taking a major role in the pathophysiology. Accordingly, the suggested therapeutic options for this disease include elimination diets, steroids, and antieosinophil medications. Unfortunately, those treatment options are limited by their efficacy and the side effects associated with their chronic use. In the current review, we describe the characteristics of eosinophilic esophagitis and present the available treatments and their pitfalls. We also speculate on the future development of new treatment for this chronic disease.
\end{abstract}

Keywords: eosinophilic esophagitis, treatment, children, review

\section{Introduction}

Eosinophilic esophagitis (EoE) has been defined as a clinicopathologic disorder characterized by symptoms related to esophageal dysfunction and pathologically with eosinophil-predominant inflammation affecting adults and children. ${ }^{1,2}$ The nonspecific definition of this disease is the result of clinical symptoms with characteristic histological findings in the esophagus, but with no specific pathophysiological mechanism described. Clinically the disease can present in children with characteristic symptoms; ie, dysphagia or food impaction, but may also be presented with various symptoms such as abdominal pain, vomiting, reflux related symptoms, and failure to thrive in young children. The clinical symptoms may vary from patient to patient and may change with increasing age..$^{3-5}$ To date, there are no clinical features specific enough to make the diagnosis of EoE. ${ }^{1}$ Interestingly, allergies (food or environmental) have been described in most of the patients with this disease, suggesting a possible etiology for EoE. Nonetheless, many confounding factors that may influence the clinical presentation and prognosis of this disease were also described including: environmental, ethnic, genetic, and possibly other yet unknown factors. ${ }^{6}$ Endoscopically, the disease
Correspondence: Yoram Elitsur John C Edwards School of Medicine, Marshall University, I 300 Medical Center Drive, Huntington, WV, USA, 25701

Tel + I 304 69| |38|

Fax + I 304 691 1375

Email elitsur@marshall.edu 
is characterized by mucosal changes (rings, furrows, white plaques, crepe mucosa, and stenosis), while the histology is characterized by a high load of eosinophils in the esophageal mucosa; however, there is no single endoscopic or histological feature that accurately establishes the diagnosis. ${ }^{1,7}$ To date, the exact pathophysiology of the disease has not been revealed, although clinical and laboratory studies suggest the involvement of the mucosal immune system, especially those immune mediators that are related to the eosinophil cell lineage. ${ }^{8}$

EoE has been recognized as a chronic disease and without therapy it carries a significant morbidity including esophageal fibrosis and stenosis. As in many chronic diseases in children (eg, inflammatory bowel disease, celiac disease, etc), the prolonged therapy needed for EoE is associated with significant morbidity and life difficulties. A previous report showed a significant psychosocial impact of the disease, especially in older children. ${ }^{9}$ Another study decribed a significant worsening in quality of life. ${ }^{10}$ These side effects may have significant implications on patients' compliance, treatment success, and long term prognosis for children with EoE.

\section{Therapy and future therapeutic options}

For lack of specific etiology, all therapeutic options aim towards the resolution of the clinical symptoms and/or the healing of the esophageal mucosa. Unfortunately, EoE is considered to be a chronic disease and as such, therapy is indefinite with many patients experiencing episodic cycles of remissions and exacerbations. Previous data in adults and children suggest that without therapy the long term outcome will result in esophageal stenosis and therapy will prevent those complications. ${ }^{11}$ As food allergy is recognized as one possible etiology for EoE, eliminating food allergens is the first therapeutic option for these patients. Unfortunately, this option is unfriendly to children, unsustainable for the long term, and may cause nutrient and vitamin deficiencies for growing children. Other therapeutic options, ie, immunosuppressive drugs (steroids, anti-EoE modulators), carry the potential for the development of significant side effects such as esophageal candidiasis and/or other metabolic and endocrine side effects. In the current review, the available therapies for EoE and their pitfalls will be discussed.

\section{Dietary treatment}

Eosinophilic esophagitis has been associated with food and environmental allergies. In fact, in the initial description of the disease, food was implicated as one possible etiology. ${ }^{12}$
Previous reports showed that over $90 \%$ of children with EoE may have food or environmental allergies..$^{1,2,11,13}$ Accordingly, one of the earliest prescribed treatments was the elimination of food allergens from the diet ("the elimination diet"). ${ }^{12}$ Indeed, whenever a food allergy was detected, the elimination of those allergens was found to be successful in resolving the patient's symptoms and healing the esophageal pathology (decreased esophageal eosinophil counts and submucosal fibrosis). ${ }^{14-17}$ The logic behind this therapy may seem straightforward, but others showed that even without any food allergy testing, and/or detection of food allergens by testing, a six-food elimination diet was found to efficiently resolve symptoms and to heal esophageal mucosal pathology. ${ }^{17,18}$

As dietary elimination protocols have not been standardized, different dietary treatment options are used. The most common protocols included elimination of the most common six food allergens, elimination of foods identified by allergy testing (positive skin prick and patch tests), elimination of the three most common food allergens (milk, egg, and wheat), a vegan diet, and even one food elimination (milk). ${ }^{1,19,20}$ Spergel et al reported in their retrospective review of 941 children with EoE, at the Children's Hospital of Philadelphia, that the elimination dietary protocols with the best success rates $(77 \%)$ were those which were directed by allergy testing plus milk elimination. ${ }^{19}$ The lack of head-tohead comparison between the different protocols in children prevents us from declaring the optimal protocol for children with EoE; thus, all the success rates reported by different authors represent their own studies only. Overall, the literature has described different elimination diet protocols that were reported to be effective for EoE, but the lack of standardization and the missing "cause and effect" reason is confusing.

The success of some dietary protocols without proven food allergens has not been fully explained. The allergy experts implicated the technical limitations of the allergy testing. They suggested that all our current tests to assess food allergy (immunoassay, skin prick, or atopy patch test) have a limited accuracy rate, and suggested that the combination of two allergy tests (skin prick and atopy patch test) may improve the accuracy of these tests. ${ }^{21}$ Others explained it by the lack of standardization among centers in regard to antigen types, technical differences in skin testing, and/or read out of results. ${ }^{11}$ It is clear that avoidance of food items improves clinical and histological pathology, but the exact mechanism has yet to be determined.

In addition to the lack of dietary protocol standardization, food elimination therapy may not be practical for young patients and their families. Some of the most common food 
allergens (milk, soy, egg, wheat, tree nuts/peanuts, fish/ shellfish) are crucial for children's normal growth and development. Accordingly, replacement of these food items is needed and may be costly and time consuming for the parents. Moreover, as children are growing, the dietary limitation becomes impossible to implement for long periods of time. Unfortunately, most of the prospective studies on diet therapy for EoE in children are short term and do not answer the feasibility of this therapy for the long term ( $>1$ year). The low compliance rate of dietary treatment documented in adults with EoE and the low quality of life under dietary restriction reported in children may serve as a reality check for the feasibility of these therapies. ${ }^{10,22}$

Lastly, in spite of the close association between food allergy and EoE, in a large retrospective cohort study, food allergens were detected in only one third of the children, suggesting that food elimination treatment directed by allergy testing would not cover all EoE patients. ${ }^{21}$

The importance of environmental allergens in the pathophysiology of EoE has not been fully explored or explained. Environmental allergens are commonly associated with EoE in children and adults and were reported in over $70 \%$ of those patients. ${ }^{1,13}$ Studies on animal models and humans have suggested their pathophysiological role in this disease, but there are no good prospective studies that specifically addressed this topic in children or adults. ${ }^{6,23}$ In a retrospective study, allergic asthma and peripheral eosinophils were predictive variables for EoE in adults with asthma. ${ }^{24}$ When considering all other allergy related diseases in children (ie, allergic rhinitis, eczema, allergic conjunctivitis, etc), it becomes clear that different allergens (food and environmental) are participating in the pathophysiology of EoE, but the exact relationship and their contribution to the disease's pathophysiology is yet unknown.

\section{Drug therapy}

The recommended drug for the treatment for EoE in children is steroids. Steroids in their various forms (topical and systemic) have shown a good efficacy in reducing esophageal inflammation, reducing eosinophil load, and reversing fibrosis accumulation. ${ }^{25,26}$ To minimize the side effects of systemic steroids, topical steroids are the recommended first line of therapy, followed by systemic steroids if no improvement is achieved. Topical steroids are divided into inhaled steroids (budesonide, fluticasone) which is sprayed on the tongue twice (bid) or three (tid) times per day, or "steroid slush" which is a liquid steroid compound (beclomethasone respules) with added sugar for the child to drink. The last option is used for younger children where the spray method is not feasible. The basic assumption for this mode of therapy is that topical steroids are absorbed in the esophageal mucosa and help to resolve the inflammation and to decrease eosinophil infiltration.

The effect of steroid therapy in EoE for children has been proven in many reports. ${ }^{1,27,28}$ Moreover, in a head-to-head comparison, topical steroids were as effective as systemic steroids, but without the systemic steroids' side effects. ${ }^{29}$ Nevertheless, in a double-blind, placebo-controlled study in adults, Alexander et al showed that symptoms were not better after steroid therapy compared to placebo, suggesting that there are other, yet unknown factors that influenced the treatment's success. ${ }^{30}$

Indeed, the pharmacokinetics of topical steroid therapy has never been evaluated in children. Considering that the esophageal mucosa was never considered as an absorptive organ in the human body, it is quite surprising that the spray method works at all in children. Moreover, the squamous cell epithelium and the physiological epithelial keratinization process may further block any absorption of drugs. Using nuclear scintigraphy techniques, Dellon et al showed that the mucosal contact time of the viscous form of steroid was longer compared to the nebulized form; however, the scanning also showed that the medications reached the gastric mucosa within seconds, suggesting that esophageal mucosal contact time with the drug is limited and absorption is very unlikely or even impossible. ${ }^{31}$ Moreover, a large quantity of the nebulized steroids was detected in the lungs, suggesting an inefficient delivery of the drug to the esophagus. It is thus surprising that in spite of the inefficient delivery of the nebulized (topical) steroid and the proven absorption of systemic steroids (confirmed by serum level and clinical side effects), the latter were not superior to topical steroids. ${ }^{28,29}$ Overall, it is clear that the pharmacodynamics of the topical steroids in children with EoE is unknown, and whether the drug has a topical or systemic effect on the esophagus is yet to be explored.

\section{Non-steroid therapy Monoclonal antibodies}

The involvement of the immune system and its crucial participation in the pathophysiology of EoE is described elsewhere and is not the topic of this review. ${ }^{1,8}$ The development of the eosinophil cell lineage is influenced by many immune factors including lymphokines, cytokines, and many others. Interleukin 5 (IL-5) and eotaxin are two major immune modulators affecting eosinophil proliferation and are elevated in children with EoE. ${ }^{1,8}$ Accordingly, using pharmacological 
blockers for those modulators was suggested as the next option for therapy. ${ }^{32}$ Indeed, in another eosinophilic related disease, hypereosinophilic syndrome, monoclonal anti-IL-5 (mepolizumab) significantly reduced the number of eosinophils and is now considered as a potential therapy for this disease. ${ }^{33}$ The therapeutic trials of this medication in children and adults with EoE were less successful. ${ }^{11,34-36}$ In a multicenter, blinded, controlled pediatric study, Ass'ad et al showed that mepolizumab reduced eosinophil counts, but symptoms were not significantly better compared to placebo. ${ }^{35}$ The high cost and low efficacy of mepolizumab limited its use as a therapy for children with EoE. In other studies, different monoclonal antibodies (reslizumab, omalizumab) did not show any promising results. ${ }^{34,36}$ In summary, the use of specific antibodies against various immune mediators participating in the pathophysiology of EoE are not ready yet for prime time.

\section{Proton-pump inhibitor (PPI) therapy}

In the past, the presence of eosinophils in the esophageal mucosa was considered as one of the classic markers for gastroesophageal reflux disease (GERD) in children and antacid medications ( $\mathrm{H}_{2}$ blockers, PPIs) were the accepted therapy for this disease. The high load of eosinophils in the esophagus of patients led physicians to widen their differential diagnosis. In the beginning, the high number of esophageal eosinophils (15 eosinophils per high powered field) was considered as one of the discriminatory markers separating EoE from GERD. Nevertheless, this marker lost some of its specificity once Ngo et al and others described children with GERD who had comparable numbers of eosinophils and positive $\mathrm{pH}$ study. ${ }^{37,38-42}$ More importantly, over $40 \%$ of children with high eosinophil counts will respond to PPI therapy (symptom resolution and decreased eosinophils infiltration), but no clinical or histological predictors could differentiate between responders and nonresponders. ${ }^{39}$ Those responders were later defined as patients with PPI responsive EoE (PPIREE). ${ }^{11,43}$

The relationship between GERD, EoE, and PPIREE is not well defined. The previous assumption that GERD will respond to PPI therapy but not to EoE failed clinical experience and the new diagnosis (PPIREE) has now shaken this dichotomy. Accordingly, the prerequisite of a normal $\mathrm{pH}$ study before the diagnosis of EoE could be established has lost some of its legitimacy as PPIREE may present with a normal or abnormal $\mathrm{pH}$ study. ${ }^{38,40}$ Consequently, the request for patients suspected of EoE to fail PPI therapy first followed by endoscopic confirmation became expensive and may not be practical or needed. The new defined disease (PPIREE) can be presented with clinical or histological features of both diseases (GERD, EoE), a situation that may raise confusion and many unanswered questions. For example, what is the long term prognosis of PPIREE? Could EoE patients be camouflaged as GERD (a wolf in a sheep's clothing)? Is PPIREE an intermediate step before EoE develops? In support of that hypothesis, investigators showed that in the long term, PPIREE patients may lose their response to PPI and will need steroid treatment. ${ }^{40,42}$

To date, the pathophysiology of PPIREE is unknown. A few investigators suggested that acid reflux irritates the esophageal mucosa to increase permeability and allow foreign antigens to stimulate the recruitment of eosinophils. ${ }^{44-46}$ In cell culture studies, PPI was shown to reduce stimulation of several cytokines that are closely related to eosinophil physiology (interleukin 6, tumor necrosis factor, eotaxin-3). ${ }^{47,48}$ In an esophageal squamous cell line derived from biopsies of patients with EoE, Zhang et al showed that omeprazole's (PPI) anti-eosinophil effect is caused by blocking the binding of signal transducer and activator of transcription 6 to the eotaxin-3 promoter. ${ }^{49}$ This data might help explain the therapeutic effect of PPI on eosinophil recruitment, but if we consider this hypothesis to be correct, one would expect a much higher percentage of PPIREE disease in patients with GERD. Hirano estimated that in the world of eosinophilic related esophageal diseases, PPIREE is a very small percentage of the total GERD patients and is divided approximately equally between PPIREE EoE related, PPIREE GERD related, and PPIREE non-EoE and non-GERD related diseases. ${ }^{43}$

Currently, PPI is the recommended therapy for PPIREE but there are no prospective studies to support the dose, type, or frequency of medications used in children. How long should we continue with PPI? What is the role of $\mathrm{H}_{2}$ blockers in this disease? What is the needed frequency of upper endoscopy to follow those patients? In the recently published clinical guideline, it was recommended that PPI (any type) should be used twice a day until better information is available. ${ }^{11}$

In summary, PPIREE disease in the spectrum of GERD and EoE has not been clearly defined. The relationship between those diseases is not known or scientifically explained. The coexistence of those diseases needs to be explored, and a combined therapy with both PPI and steroids, as practiced in many clinics, may be a reasonable approach until further information is available. ${ }^{3}$

\section{Goal of therapy}

In spite of various experts' clinical guidelines, there are significant differences in the clinical treatment protocols and 
follow up for children diagnosed with EoE. Some experts recommended targeting eosinophil counts as the goal of therapy, while most practitioners will follow the symptoms as their guideline. Unfortunately both therapeutic goals suffer from significant flaws. Previous studies showed that except for dysphagia or food impaction, symptoms are not a good indicator for the disease's remission. ${ }^{7,11}$ On the other hand, following eosinophil counts requires repeated endoscopic procedures which are expensive and unacceptable for many parents. Recently, several investigators suggested using the eosinophil degranulation proteins (Myc promoter-binding protein-1, Eosinophilic cationic protein, eosinophil-derived neurotoxin, eosinophil primary granule) as tissue biomarkers for clinical follow up. ${ }^{50,51}$ Eosinophil "debris," collected from the esophagus by a string, is reported to be a specific marker for mucosal inflammation. ${ }^{52}$ Unfortunately, the string test has not been standardized and is still under investigation. The proper way to address the "goal of therapy" is still under discussion and a proven guideline is clearly needed.

\section{Future treatment development for children with EoE}

Since the discovery of EoE, its clinical or histological diagnosis became murkier. The disease's pathophysiology is not clear and the therapeutic results of the recommended drugs have been disappointing so far. It is clear that the chronicity of EoE increased the disease's morbidity and diminished its clinical prognosis. To date, investigators have described three overlapping diseases (GERD, EoE, PPIREE) with no good demarcation among them in regards to clinical symptoms, pathophysiology, or treatment. It is well-accepted that those diseases are related to each other and are controlled by many confounding factors including age, gender, race, genes, allergies, and other yet unknown factors. The current main therapy available for EoE is directed to reverse the histopathology described in the esophagus (tissue inflammation and eosinophil infiltration) and improve patient symptoms. Elimination diet and steroids are the current prescribed treatments. Unfortunately, in placebo-controlled studies, the clinical results of those steroid medications were limited, mainly due to the unbearable side effects of chronic steroid therapy. Unfortunately, following our experience with inflammatory bowel disease, it is predicted that the current drug armamentarium will eventually fail and newer more potent drugs will be needed. It seems that without finding the holy grail of the disease (ie, celiac disease), we will keep going in circles without a real sustained solution. In short, in order to provide long-standing remission and possibly full recovery from the disease, research should address the pathophysiology of the disease rather than its symptoms or histology. ${ }^{53}$ The new active research targeting the immune regulation of the eosinophil lineage may shed light on the pathophysiology of EoE, but the multifactorial etiologies already known in this disease (environment, genetics, race, etc) suggest that finding the holy grail is not likely and that EoE pathophysiology would follow the clinicopathologic pathway of inflammatory bowel disease.

\section{Disclosure}

The author reports no conflicts of interest in this work.

\section{References}

1. Liacouras CA, Furuta GT, Hirano I, et al. Eosinophilic esophagitis: Updated consensus recommendations for children and adults. JAllergy Clin Immunol. 2011;128(1):3-20. e6; quiz 21.

2. Furuta GT, Liacouras CA, Collins MH, et al; First International Gastrointestinal Eosinophil Research Symposium (FIGERS) Subcommittees. Eosinophilic esophagitis in children and adults: a systemic review and consensus recommendations for diagnosis and treatment. Gastroenterology. 2007;133(4):1342-1363.

3. Levine J, Lai J, Edelman M, Schuval SJ. Conservative long-term treatment of children with eosinophilic esophagitis. Ann Allergy Asthma Immunol. 2012;108(5):363-366.

4. Straumann A, Aceves SS, Blanchard C, et al. Pediatric and adult eosinophilic esophagitis: similarities and differences. Allergy. 2012;67(4):477-490.

5. Pentiuk S, Putnam PE, Collins MH, Rothenberg ME. Dissociation between symptoms and histological severity in pediatric eosinophilic esophagitis. J Pediatr Gastroenterol Nutr. 2009;48(2):152-160.

6. Elitsur Y. Confounding factors affect the pathophysiology of eosinophilic esophagitis. World J Gastroenterol. 2012;18(33): 4466-4469.

7. Al-Subu A, Bevins L, Yulia D, Elitsur Y. The accuracy of endoscopic features in eosinophilic esophagitis: the experience in children from rural West Virginia. J Clin Gastroenterol. 2012;46(10):e83-e86.

8. Blanchard C, Wang N, Rothenberg ME. Eosinophilic esophagitis: pathogenesis, genetics, and therapy. J Allergy Clin Immunol. 2006;118(5):1054-1059.

9. Harris RF, Menard-Katcher C, Atkins D, Furuta GT, Klinnert MD. Psychosocial dysfunction in children and adolescents with eosinophilic esophagitis. J Pediatr Gastroentrol Nutr. Epub June 6, 2013.

10. Franciosi JP, Hommel KA, Bendo CB, et al. PedsQL ${ }^{\text {TM }}$ eosinophilic esophagitis module: feasibility, reliability and validity. $J$ Pediatr Gastroenterol Nutr. 2013;57(1):57-66.

11. Dellon ES, Gonsalves N, Hirano I, Furuta GT, Liacouras CA, Katzka DA; American College of Gastroenterology. ACG clinical guideline: Evidenced based approach to the diagnosis and management of esophageal eosinophilia and eosinophilic esophagitis (EoE). Am J Gastroenterol. 2013;108(5):679-692; quiz 693.

12. Kelly KJ, Lazenby AJ, Rowe PC, Yardley JH, Perman JA, Sampson HA. Eosinophilic esophagitis attributed to gastroesophageal reflux: improvement with an amino acid-based formula. Gastroenterology. 1995;109(5):1503-1512.

13. Otteson TD, Mantle BA, Casselbrant ML, Goyal A. The otolaryngologic manifestations in children with eosinophilic esophagitis. Int J Pediatr Otorhinolaryngol. 2012;76(1):116-119.

14. Kagalwalla AF, Sentongo TA, Ritz S, et al. Effect of six-food elimination diet on clinical and histologic outcomes in eosinophilic esophagitis. Clin Gastroenterol Hepatol. 2006;4(9):1097-1102. 
15. Spergel JM, Beausoliel JL, Mascarenhas M, Liacouras CA. The use of skin prick tests and patch tests to identify causative foods in eosinophilic esophagitis. J Allergy Clin Immunol. 2002;109(2):363-368.

16. Liacouras CA, Spergel JM, Ruchelli E, et al. Eosinophilic esophagitis: a 10-year experience in 381 children. Clin Gastroenterol Hepatol. 2005;3(12):1198-1206.

17. Kagalwalla AF, Shah A, Li BU, et al. Identification of specific foods responsible for inflammation in children with eosinophilic esophagitis successfully treated with empiric elimination diet. $J$ Pediatr Gastroenterol Nutr. 2011;53(2):145-149.

18. Spergel JM, Andrews T, Brown-Whitehorn TF, Beausoleil JL, Liacouras CA. Treatment of eosinophilic esophagitis with specific food elimination diet directed by a combination of skin prick and patch tests. Ann Allergy Asthma Immunol. 2005;95(4):336-343.

19. Spergel JM, Brown-Whitehorn T, Cianferoni A, et al. Identification of causative food in children with eosinophilic esophagitis treated with an elimentation diet. J Allergy Clin Immunol. 2012;130(2):461-467. e5.

20. Kagalwalla AF, Amsden K, Shah A, et al. Cow's milk elimination: a novel dietary approach to treat eosinophilic esophagitis. J Pediatr Gastroenterol Nutr. 2012;55(6):711-716.

21. Gonsalves N, Yang GY, Doerfler B, Ritz S, Ditto AM, Hirano I. Elimination diet effectively treats eosinophilic esophagitis in adults; food reintroduction identifies causative factors. Gastroenterology. 2012;142(7):1451-1459. e1; quiz e14.

22. Peterson KA, Byrne KR, Vinson LA, et al. Elemental diet induces histologic response in adult eosinophilic esophagitis. Am J Gastroenterol. 2013;108(5):759-766.

23. Mishra A, Hogan SP, Brandt EB, Rothenberg ME. An etiological role for aeroallergens and eosinophils in experimental esophagitis. J Clin Invest. 2001;107(1):83-90.

24. Harer KN, Enders FT, Lim KG, Alexander JA, Katzka DA. An allergic phenotype and the use of steroid inhalers predict eosinophilic oesophagitis in patients with asthma. Aliment Pharmacol Ther. 2013;37(1):107-113.

25. Abu-Sultaneh SM, Durst P, Maynard V, Elitsur Y. Fluticasone reversed sub-epithelial fibrosis in children with eosinophilic esophagitis. Dig Dis Sci. 2011;56(1):97-102.

26. Aceves SS, Newbury RO, Chen D, et al. Resolution of remodeling in eosinophilic esophagitis correlates with epithelial response to topical corticosteroids. Allergy. 2010;65(1):109-116.

27. Dohil R, Newbury R, Foz L, Bastian J, Aceves S. Oral viscous budenoside is effective in children with eosinophilic esophagitis in a randomized, placebo controlled trial. Gastroenterology. 2010;139(2): 418-429.

28. Knoikoff MR, Noel RJ, Blanchard C, et al. A randomized, doubleblind, placebo-controlled trial of fluticasone propionate for pediatric eosinophilic esophagitis. Gastroenterology. 2006;131(5):1381-1391.

29. Schafer ET, Fitzgerald JF, Molleston JP, et al. Comparison of oral prednisone and topical fluticasone in the treatment of eosinophilic esophagitis: a randomized trial in children. Clin Gastroenterol Hepatol. 2008;6(2):165-173.

30. Alexander JA, Jung KW, Arora AS, et al. Swallowed fluticasone improves histologic but not symptomatic responses of adults with eosinophilic esophagitis. Clin Gastroenterol Hepatol. 2012;10(7): 742-749. e1.

31. Dellon ES, Sheikh A, Speck O, et al. Viscous topical is more effective than nebulized steroid therapy for patients with eosinophilic esophagitis. Gastroenterology. 2012;143(2):321-324. e1.

32. Garrean C, Hirano I. Eosinophilic esophagitis: pathophysiology and optimal management. Curr Gastroenterol Rep. 2009;11(3):175-181.

33. Garrett JK, Jameson SC, Thompson B, et al. Anti-interleukin-5 (mepolizumab) therapy for hypereosinophilic syndrome. J Allergy Clin Immunol. 2004;113(1):115-119.

34. Spergel JM, Rothenberg ME, Collins MH, et al. Reslizumab in children and adolescents with eosinophilic esophagitis: Results of a doubleblind, randomized, placebo-controlled trial. J Allergy Clin Immunol. 2012;129(2):456-463, 463. e1.
35. Ass'ad AH, Gupta SK, Collins MH, et al. An antibody against IL-5 reduces the numbers of esophageal intraepithelial eosinophils in children with eosinophilic esophagitis. Gastroenterology. 2011;141(5): 1593-1604.

36. Rocha R, Vitor AB, Trindade E, et al. Omalizumab in the treatment of eosinophilic esophagitis and food allergy. Eur J Pediatr. 2011;170(11): 1471-1474.

37. Ngo P, Furuta GT, Antonioli DA, Fox VL. Eosinophils in the esophagus - peptic or allergic eosinophilic esophagitis? Case series of three patients with esophageal eosinophilia. Am J Gastroenterol. 2006;101(7):1666-1670.

38. Molina-Infante J, Ferrando-Lamana L, Ripoll C, et al. Esophageal eosinophilic infiltration responds to proton pump inhibition in most adults. Clin Gastroenterol Hepatol. 2011;9(2):110-117.

39. Dranove JE, Horn DS, Davis MA, Kernek KM, Gupta SK. Predictors of response to proton pump inhibitor therapy among children with significant esophageal eosinophilia. J Pediatr. 2009;154(1):96-100.

40. Sayej WN, Patel R, Baker RD, Tron E, Baker SS. Treatment with highdose proton pump inhibitors helps distinguish eosinophilic esophagitis from noneosinophilic esophagitis. J Pediatr Gastroenterol Nutr. 2009;49(4):393-399.

41. Dohil R, Newbury RO, Aceves S. Transient PPI responsive esophageal eosinophilia may be a clinical sub-phenotype of pediatric eosinophilic esophagitis. Dig Dis Sci. 2012;57(5):1413-1419.

42. Schroeder S, Capocelli KE, Masterson JC, et al. Effect of proton pump inhibitor on esophageal eosinophilia. J Pediatr Gastroenterol Nutr. 2013;56(2):166-172.

43. Hirano I. Editorial: Should patients with suspected eosinophilic esophagitis undergo a therapeutic trial of proton pump inhibition? Am J Gastroenterol. 2013;108(3):373-375.

44. Moawad FJ, Veerappan GR, Dias JA, Baker TP, Maydonovitch CL, Wong RK. Randomized controlled trial comparing aerosolized swallowed fluticasone to esomeprazole for esophageal eosinophilia. Am J Gastroenterol. 2013;108(3):366-372.

45. Spechler SJ, Genta RM, Souza RF. Thoughts on the complex relationship between gastroesophgeal reflux disease and eosinophilic esophagitis. Am J Gastroenterol. 2007;102(6):1301-1306.

46. Tobey NA, Carson JL, Alkiek RA, Orlando RC. Dilated intercellular spaces: a morphological feature of acid reflux - damaged human esophageal epithelium. Gastroenterology. 1996;111(5):1200-1205.

47. Kedika RR, Souza RF, Spechler SJ. Potential anti-inflammatory effects of proton pump inhibitors: A review and discussion of the clinical implications. Dig Dis Sci. 2009;54(11):2312-2317.

48. Cheng E, Zhang X, Huo X et al. Omeprazole blocks eotaxin-3 expression by esophageal squamous cells from patients with eosinophilic esophagitis and GORD. Gut. 2013;62(6):824-832.

49. Zhang X, Cheng E, Huo X, et al. Omeprazole blocks STAT6 binding to the eotaxin-3 promoter in eosinophilic esophagitis cells. PLOS ONE. 2012;7(11):e50037.

50. Mueller S, Neureiter D, Aigner T, Stolte M. Comparison of histological parameters for the diagnosis of eosinophilic oesophagitis versus gastrooesophageal reflux disease on oesophageal biopsy material. Histopathology. 2008;53(6):676-684.

51. Dellon ES, Chen X, Miller CR, Woosley JT, Shaheen NJ. Diagnostic utility of major basic protein, eotaxin-3, and leukotriene enzyme staining in eosinophilic esophagitis. Am J Gastroenterol. 2012;107(10): 1503-1511.

52. Furuta GT, Kagalwalla AF, Lee JJ, et al. The esophageal string test: a novel, minimally invasive method measures mucosal inflammation in eosinophilic esophagitis. Gut. Epub August 15, 2012.

53. Rothenberg ME. Biology and treatment of eosinophilic esophagitis. Gastroenterology. 2009;137(4):1238-1249. 
Pediatric Health, Medicine and Therapeutics

Dovepress

\section{Publish your work in this journal}

Pediatric Health, Medicine and Therapeutics is an international, peerreviewed, open access journal publishing original research, reports, editorials, reviews and commentaries. All aspects of health maintenance, preventative measures and disease treatment interventions are addressed within the journal. Practitioners from all disciplines are invited to submit their work as well as healthcare researchers and patient support groups. The manuscript management system is completely online and includes a very quick and fair peer-review system. Visit http://www.dovepress.com/ testimonials.php to read real quotes from published authors.

Submit your manuscript here: http://www.dovepress.com/pediatric-health-medicine-and-therapeutics-journal 